\title{
Bayesian Analysis of Rayleigh Distribution Under Quasi-Prior for Different Loss Functions
}

\author{
Paresh Sanat $^{1}$, R. S. Srivastava ${ }^{2}$ \\ ${ }^{1,2}$ Department of Mathematics and Statistics, D. D. U. Gorakhpur University, Gorakhpur, 273009, India
}

\begin{abstract}
The Rayleigh distribution has a wide range of applications in fields such as survival analysis, life testing, reliability theory, clinical studies and communication engineering. This distribution is also used in some problem of physics and engineering, such as the diffusion process or the distribution of the power (or amplitude) of electromagnetic waves received through a scattering medium. In this paper, we consider the estimator of the parameter of Rayleigh distribution. Bayes estimators are obtained by using Quasi prior under squared error loss function, precautionary loss function and another loss function. Maximum likelihood estimator is also obtained.
\end{abstract}

Keywords: Rayleigh distribution, Quasi prior, squared error loss function, precautionary loss function and another loss function, MLE etc

\section{Introduction}

The Rayleigh distribution is often observed when the overall magnitude of a vector is related to its directional components. One example, where the Rayleigh distribution naturally arises when velocity is analyzed into its orthogonal 2-dimensional components. This distribution is considered to be a very useful life time distribution. It is an important distribution for operation research and statistical fields. It is used in many areas such as health, agriculture, biology, and other sciences. One of the most important appliciation of this stochastic model is used in analyzing wind speed data. Two parameter Weibull distribution with the shape parameter equal to 2 is referred to as Rayleigh distribution. This model was first introduced by Lord Rayleigh (1880). Siddiqui (1962) discussed the origin and properties of the Rayleigh distribution. Inference for Rayleigh model has been considered by Sinha and Howlader (1993), Mishra et al. (1996) and Abd Elfattah et al. (2006).

The probability density function of Rayleigh distribution is given as:

$$
\mathrm{f}(\mathrm{y} ; \theta)=\frac{y}{\theta^{2}} \exp \left(-\frac{y^{2}}{2 \theta^{2}}\right) ; \mathrm{y} \geq 0, \theta>0
$$

Bayesian analysis is an important approach to statistics, which formally seeks use of prior information and Bayes theorem provides the formal basis for using this information. There is no clear way that which prior is better than the other. Often priors are chosen accordingly to subjective knowledge and beliefs. However, if one has adequate information about parameters one should use informative prior(s); otherwise it is preferable to use non-informative prior(s). Ahmed A. et al. (2013).

The choice of a prior distribution and loss function is an integral part of Bayesian inference. As there is no specific procedure that allows us to identify the appropriate loss function to be used, the choice of loss function depends on the seriousness of errors i.e., over estimation or under estimation. The squared error loss function (SELF) is appropriate when over and under estimations both are equally serious. Otherwise one has to use asymmetric loss functions. For example, Linex, Precautionary and some other loss functions which are not symmetric. Varian (1975),
Zellner (1986), Chandra (2001), Norstrom J.G.(1996), Srivastava R.S. and Joshi N.K.(2004), Srivastava R.S. and Singh S.P.(2007), Srivastava R.S. and Rao A.K.(2007) etc. In this paper, we consider the above loss functions for better comparison of Bayesian analysis.

a) The first is the common squared error loss function given by:

$$
l_{1}(\widehat{\theta}, \theta)=(\hat{\theta}-\theta)^{2}
$$

which is symmetric, $\theta$ and $\hat{\theta}$ represent the true and estimated values of the parameter. This loss function is frequently used because of its analytical tractability in Bayesian analysis.

b) The second is the precautionary loss function given by:

$$
l_{2}(\widehat{\theta}, \theta)=\frac{(\widehat{\theta}-\theta)^{2}}{\widehat{\theta}}
$$

which is asymmetric, $\theta$ and $\hat{\theta}$ represent the true and estimated values of the parameter. This loss function is used when the under estimation is more serious consequences.

c) The third loss function is given by:

$$
l_{3}(\widehat{\theta}, \theta)=\frac{(\widehat{\boldsymbol{\theta}}-\boldsymbol{\theta})^{2}}{\boldsymbol{\theta}^{\mathbf{2}}}
$$

which is asymmetric, $\theta$ and $\hat{\theta}$ represent the true and estimated values of the parameter.

\section{Maximum likelihood estimation}

Maximum likelihood estimation of the parameter of Rayleigh distribution is well discussed in literature (Cohen, (1965) and Mann et al. (1975)).

Let $\underline{y}=\left(y_{1}, \ldots, y_{n}\right)$ be a random sample of size $\mathrm{n}$ having the probability density function as

$$
\mathrm{f}(\mathrm{y} ; \theta)=\frac{y}{\theta^{2}} \exp \left(-\frac{y^{2}}{2 \theta^{2}}\right) ; \mathrm{y} \geq 0, \theta>0
$$

The M. L. Estimator of $\theta$ is

$$
\widehat{\Theta}=\sqrt{\frac{\sum_{1}^{n} y_{i}^{2}}{2 n}}
$$

\section{Bayesian estimation of Rayleigh distribution under Quasi prior by using Squared Error Loss Function}

Consider there are $\mathrm{n}$ recorded values, $\underline{y}=\left(y_{1}, \ldots, y_{n}\right)$ from (1.1). We consider the Quasi prior as

$$
g(\theta)=\frac{1}{\theta^{d}}
$$




\section{International Journal of Science and Research (IJSR) \\ ISSN (Online): 2319-7064}

Index Copernicus Value (2013): 6.14 | Impact Factor (2015): 6.391

the pdf of Rayleigh distribution is

$$
\mathrm{f}(\mathrm{y} ; \theta)=\frac{y}{\theta^{2}} \exp \left(-\frac{y^{2}}{2 \theta^{2}}\right) ; \mathrm{y} \geq 0, \theta>0
$$

the likelihood function is given by

$$
\mathrm{L}(\mathrm{y} \mid \theta)=\frac{\prod_{1}^{n} y_{i}}{\theta^{2 n}} e^{\left(-\frac{\sum_{1}^{n} y_{i}^{2}}{2 \theta^{2}}\right)}
$$

the posterior density function is given by

$\mathrm{P}(\theta \mid y)=\mathrm{L}(\mathrm{y} \mid \theta) \mathrm{g}(\theta)$

then the posterior density of $\theta$ is by

$$
\mathrm{P}(\theta \mid \underline{y}) \propto \frac{\prod_{1}^{n} y_{i}}{\theta^{2 n+d}} e^{\left(-\frac{\sum_{1}^{n} y_{i}^{2}}{2 \theta^{2}}\right)}
$$

Estimation under squared error loss function (SELF):

By using a squared error loss function

$$
\mathrm{L}(\widehat{\theta}, \theta)=(\widehat{\theta}-\theta)^{2}
$$

The posterior risk function is

$$
\mathrm{R}(\hat{\theta})=\int_{0}^{\infty}(\hat{\theta}-\theta)^{2} P(\theta \mid \underline{y}) \mathrm{d} \theta
$$

$\mathrm{R}(\hat{\theta})=\hat{\theta}^{2} \prod_{1}^{n} y_{i} \Gamma \frac{2 n+d-1}{2}$

$\frac{\prod_{1}^{n} y_{i}}{2} \Gamma \frac{2 n+d-3}{2}\left(\frac{\sum_{1}^{n} y_{i}^{2}}{2}\right)^{\frac{3-2 n-d}{2}}$

- $\widehat{\theta} \prod_{1}^{n} y_{i} \Gamma \frac{2 n+d-2}{2}\left(\frac{\sum_{1}^{n} y_{i}{ }^{2}}{2}\right)^{\frac{1-2 n-d}{2}}$

Now $\frac{\partial}{\partial \theta} \mathrm{R}(\hat{\theta})=0$,

$$
\left(\frac{\sum_{1}^{n} y_{i}^{2}}{2}\right)^{\frac{1-2 n-d}{2}}+
$$

Then the Bayes Estimator is

$$
\widehat{\Theta}_{1}=\frac{I^{2 n+d-2}}{\frac{2}{I \frac{2 n+d-1}{2}}} \sqrt{\frac{\sum_{1}^{n} y_{i}^{2}}{2}}
$$

\section{Bayesian estimation of Rayleigh distribution under Quasi prior by using precautionary loss function, $c \frac{(\widehat{\theta}-\theta)^{2}}{\widehat{\theta}}$}

The posterior density of $\theta$ is given by

$$
\mathrm{P}(\underline{y})=\frac{\prod_{1}^{n} y_{i}}{\theta^{2 n+d}} e^{\left(-\frac{\sum_{1}^{n} y_{i}^{2}}{2 \theta^{2}}\right)}
$$

Estimation under precautionary loss function:

By using precautionary loss function

$$
\mathrm{L}(\widehat{\theta}, \theta)=\frac{(\widehat{\theta}-\theta)^{2}}{\widehat{\theta}},
$$

The risk function is

$$
\mathrm{R}(\hat{\theta})=\int_{0}^{\infty} \frac{(\hat{\theta}-\theta)^{2}}{\widehat{\theta}} P(\theta \mid \underline{y}) \mathrm{d} \theta
$$

$\mathrm{R} \quad(\hat{\theta})=\frac{\widehat{\theta}}{2} \prod_{1}^{n} y_{i} \Gamma \frac{2 n+d-1}{2} \quad\left(\frac{\sum_{1}^{n} y_{i}^{2}}{2}\right)^{\frac{1-2 n-d}{2}}+$

$\frac{\prod_{1}^{n} y_{i}}{2 \hat{\theta}} \Gamma \frac{2 n+d-3}{2}\left(\frac{\sum_{1}^{n} y_{i}{ }^{2}}{2}\right)^{\frac{3-2 n-d}{2}}$

- $\prod_{1}^{n} y_{i} \Gamma \frac{2 n+d-2}{2}\left(\frac{\sum_{1}^{n} y_{i}^{2}}{2}\right)^{\frac{2-2 n-d}{2}}$

Now $\frac{\partial}{\partial \theta} \mathrm{R}(\hat{\theta})=0$,

Then, the Bayes Estimator is

$$
\widehat{\Theta}_{2}=\sqrt{\frac{I \frac{2 n+d-3}{2}}{I \frac{2 n+d-1}{2}}} \sqrt{\frac{\sum_{1}^{n} y_{i}^{2}}{2}}
$$

5. Bayesian estimation of Rayleigh distribution under Quasi prior by using loss function $\frac{(\widehat{\theta}-\theta)^{2}}{\theta^{2}}$

The posterior density of $\theta$ is given by

$$
\mathrm{P}(\theta \mid \underline{y})=\frac{\prod_{1}^{n} y_{i}}{\theta^{2 n+d}} e^{\left(-\frac{\sum_{1}^{n} y_{i}^{2}}{2 \theta^{2}}\right)}
$$

Estimation under loss function $\frac{(\hat{\theta}-\theta)^{2}}{\theta^{2}}$

By using loss function

$$
\mathrm{L}(\hat{\theta}-\theta)=\frac{(\widehat{\theta}-\theta)^{2}}{\theta^{2}}
$$

The risk function is

$\mathrm{R}(\hat{\theta})=\int_{0}^{\infty} \frac{(\hat{\theta}-\theta)^{2}}{\theta^{2}} P(\theta / \underline{y}) \mathrm{d} \theta$

$\mathrm{R} \quad(\hat{\theta})=\frac{\hat{\theta}^{2}}{2} \prod_{1}^{n} y_{i} \Gamma \frac{2 n+d+1}{2} \quad\left(\frac{\sum_{1}^{n} y_{i}^{2}}{2}\right)^{\frac{-2 n-d-1}{2}}+$

$\frac{\prod_{1}^{n} y_{i}}{2} \Gamma \frac{2 n+d-1}{2}\left(\frac{\sum_{1}^{n} y_{i}{ }^{2}}{2}\right)^{\frac{1-2 n-d}{2}}$

- $\hat{\theta} \prod_{1}^{n} y_{i} \Gamma \frac{2 n+d}{2}\left(\frac{\sum_{1}^{n} y_{i}^{2}}{2}\right)^{\frac{-2 n-d}{2}}$

Now $\frac{\partial}{\partial \theta} \mathrm{R}(\hat{\theta})=0$,

Then the Bayes Estimator is

$$
\widehat{\Theta}_{3}=\frac{\Gamma^{2 n+d}}{I \frac{2 n+d+1}{2}} \sqrt{\frac{\sum_{1}^{n} y_{i}^{2}}{2}}
$$

\section{Conclusion}

In this paper, we have obtained the Bayes estimator of the parameter of Rayleigh distribution under the Quasi prior using three different loss functions. The loss functions are Squared error loss function (SELF), Precautionary loss function and another loss function.

\section{References}

[1] A. Ahmed, S.P. Ahmed and J.A. Joshi(2013).Bayesian analysis of Rayleigh distribution. Department of Statistics, University of Kashmir, Srinagar, India.

[2] ABD Elfattah, A.M; Hassan, A.S. and Ziedan, D.M.(2006). Efficiency of Maximum likelihood estimators under different censored sampling Schemes for Rayleigh distribution, Interstat.

[3] Chandra, M. J. (2001). Statistical Quality Control. CRC Press, Boca Raton.

[4] Cohen, A. C., 1965. Maximum likelihood estimation in Weibull distribution based on complete and on censored samples. Technometrics, 7: 579-588.

[5] Lalitha,S. and Mishra, A. (1996). Modified maxcimum likelihood estimation for Rayleigh distribution, Comm.Stat.Theory Methods, 25,389-401.

[6] Norstrom J.G.(1996) :"The use of precautionary loss functions in risk analysis,"IEEE Trans. Reliab.,45(3),pp.400-40.

[7] Rahul, GP. Singh and O.P. Singh, (2009). Population project OF Kerala using Bayesian methodology .Asian J. Applied Sci., 2: 402-413.

[8] Rayleigh, J. (1880). On the resultant of a large number of vibrations of the same pitch and of arbitrary phase, Philos. Mag.;10,73-78. 
[9] Siddiqui, M.M. (1962). Some problems conectede with Rayleigh distributions,J. Res. Nat. Bur. Stand. 60D, 167-174.

[10] Sinha, S.K. and Howlader, H .A. (1993). Credible and HPD intervals of the parameter and reliability of Rayleigh distribution, IEEE Trans. Reliab;32 . 217220.

[11] Srivastava R.S. and Joshi N.K.(2004). "Entropy Bayes Estimator for Reliability of Generalized Burr life time distribution with censoring", Proceeding of ISMAMS Vol. 2(2004), pp. 158-166.

[12] Srivastava R.S. and Singh S.P.(2007). Bayesian estimation of the Shape parameter of finite Range distribution using Linex loss function with type - II censoring". South East Asian J. Math. \& Math. Sc. Vol.6 No.1 (2007), pp.1-10.

[13] Srivastava R.S. and Rao A.K. (2007). "Bayesian estimation of the scale of Gamma distribution using precautionary loss function", Statistical Techniques in Life - Testing, Reliability, Sampling Theory and Quality Control, Editor: B. N. Pandey; @ ,2007, Narosa Publishing House, New Delhi, India, pp. 122 - 127.

[14] Varian, H. R. (1975). A Bayesian approach to real estate assessment, Studies in Bayesian Econometrics and Statistics in Honor of Leonard J. Savage (eds: S.E. Fienberg and A.Zellner), North-Holland, Amsterdam, 195-208.

[15] Zellner, A. (1986). Bayesian estimation and prediction using asymmetric loss function. J.Amer., Statist. Assoc., 81, 446-451.exponential distribution using simulation Ph.D. Thesis, Baghdad University, Iraq 